\section{Linfoma no Hodgkin de células grandes "B" en cavidad oral: presentación de un caso y revisión de la literatura}

\author{
Large B-cell non-Hodgkin lymphoma of \\ the oral cavity: a case report and review of \\ literature
}

\begin{abstract}
Resumen
Los linfomas no Hodgkin conforman un grupo de neoplasias malignas de origen linfoide, clasificadas, de acuerdo con su correspondiente estirpe celular, como linfomas de células B o T. En su mayoría, estas entidades, provienen de linfocitos B, siendo el más frecuente el linfoma difuso de células B grandes (DLBCL). En cavidad oral, los linfomas no-Hodgkin tienen una baja incidencia y su pronóstico, evolución clínica y respuesta a los tratamientos, dependen del subtipo presentado. Estas neoplasias pueden desarrollarse en los tejidos blandos de la cavidad oral o de forma central en los maxilares. Se reporta un paciente de 80 años con una lesión correspondiente a linfoma no Hodgkin de células B grandes en cavidad oral, que se trató con quimioterapia y radioterapia, respondiendo favorablemente al tratamiento. Asimismo, con el objetivo de describir este tipo de lesiones poco frecuentes en cavidad oral y de valorar el pronóstico que estas tienen, se realizó una revisión de la literatura. Se concluye que debido a que el pronóstico de este tipo de lesiones depende del subtipo de linfoma no Hodgkin del que se trate y de su localización anatómica resulta muy importante realizar un correcto diagnóstico, de modo que se logre aplicar el tratamiento más adecuado para cada caso.
\end{abstract}

Palabras clave: Linfoma no Hodgkin; Boca; Patología bucal (fuente: DeCS Bireme).

\section{Caso Clínico}

Ilan Vinitzky Brener 1,2,a, Valeria Traver Méndez 2,3,b, Carlos A. Carrasco Rueda ${ }^{1, a}$, Beatriz Aldape Barrios ${ }^{4, c}$

${ }^{1}$ Instituto Nacional de Enfermedades Respiratorias Ciudad de México, México.

2 Universidad Anáhuac México, Ciudad de México, México.

${ }^{3}$ Facultad de Odontología, Universidad de Barcelona, España.

${ }^{4}$ Universidad Nacional Autónoma de México, Facultad de Odontología, Ciudad de México, México.

a Especialista en Cirugía Oral y Maxilofacial.

${ }^{b}$ Cirujano Dentista.

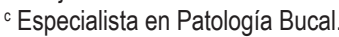

\section{Correspondencia:}

llan Vinitzky Brener: ilanvinitzky@hotmail.com Av. Ejército Nacional No. 1112 Int 1001. Colonia Los Morales Polanco, Delegación Miguel Hidalgo, Ciudad de México. México. C.P: 11510

ORCID: 0000-0001-8378-1444

\section{Coautores:}

Valeria Traver Méndez: vtraver7@hotmail.com. ORCID: 0000-0001-8376-1013

Carlos A Carrasco Rueda: dr.carrascor@hotmail.com ORCID: 0000-0001-9870-5292

Beatriz Aldape Barrios: patobu1@hotmail.com ORCID: 0000-0001-6468-7815

\section{Editora:}

Marieta Petkova-Gueorguieva

Universidad Nacional Mayor de San Marcos, Perú.

Conflicto de intereses: los autores declaramos que no existe ningún tipo de conflictos de interés.

Fuente de financiamiento: autofinanciado.

Recibido: $21 / 10 / 19$

Aceptado: $27 / 03 / 20$

Publicado: 09/05/20

\begin{abstract}
Non-Hodgkin lymphomas make up a group of malignant neoplasms of lymphoid origin, according to their corresponding cell line, as B or T cell lymphomas classified. Mostly, these entities come from B lymphocytes, being the most frequent diffuse large B-cell lymphoma (DLBCL). In the oral cavity, non-Hodgkin lymphomas have low incidence and their prognosis, clinical evolution and response to treatments, depend on the subtype presented. These malignancies can develop in soft tissues of the oral cavity or centrally in the jaws. We report an 80-year-old patient with an injury corresponding to large B-cell non-Hodgkin lymphoma in the oral cavity, who has been treated with chemotherapy and radiotherapy, responding favorably to treatment. Likewise, in order to describe this infrequent type of lesions of the oral cavity and to assess the prognosis they have, a literature review was performed. It is concluded that because of the prognosis of this type of lesions
\end{abstract}

(c) Los autores. Este artículo es publicado por la revista Odontología Sanmarquina de la Facultad de Odontología, Universidad Nacional Mayor de San Marcos. Este es un artículo de acceso abierto, distribuido bajo los términos de la licencia Creative Commons Atribucion - No Comercia_Compartir Igual 4.0 Internacional. (http://creativecommons.org/licenses/by-nc-sa/4.0/) que permite el uso no comercial, distribución y reproducción en cualquier medio, siempre que la obra original sea debidamente citada. 
depends on the subtype of non-Hodgkin lymphoma in question and its anatomical location, it is very important to make a correct diagnosis, in order to perform the adequate treatment for each case.

Keywords: Lymphoma, Non-Hodgkin; Mouth; Pathology, oral (source: MeSH NLM).

\section{Introducción}

Los linfomas no Hodgkin (LNH), son neoplasias de origen linfoide, con proliferación descontrolada. Su localización es linforreticular, pudiendo encontrase en los nódulos linfáticos, bazo, médula ósea, hígado y aparato digestivo ${ }^{1 .}$

Existen más de 30 tipos de $\mathrm{LNH}$, aproximadamente $90 \%$ son linfomas de linfocitos "B" y se presentan 14 variedades, el otro $10 \%$ corresponde a linfoma de linfocitos $\mathrm{T}$, clasificándose como distintas entidades, en base a los datos clínicos, los hallazgos morfológicos, inmunológicos, las anormalidades citogenéticas, conducta biológica y respuesta al tratamiento variable ${ }^{1}$.

En Estados Unidos, ocupan el quinto lugar en enfermedades oncológicas, donde aproximadamente 71000 personas son diagnosticadas al año con linfoma no Hodgkin y la incidencia de la enfermedad está en aumento. Desde el año 2000 hasta 2017 los linfomas han representado la octava causa de muerte por cáncer en México ${ }^{2}$.

Los linfomas de la cavidad oral, representan un 5\% de todos los linfomas y forman por sí solos el $14 \%$ de todas las neoplasias malignas de cabeza y cuello, $97 \%$ de las mismas corresponden a LNH. La mayoría de los LNH son de celularidad precursora de linfocitos $\mathrm{B}$, siendo el subtipo más frecuente el linfoma difuso de células $\mathrm{B}$ grandes (DLBCL), seguido por linfomas de células pequeñas y el linfoma de Burkitt ${ }^{3,4}$. El $2 \%$ de todos los linfomas extra-ganglionares tienen como localización primaria los siguientes sitios de la cavidad oral: paladar, encía, lengua, mucosa bucal, piso de boca y labios. Con respecto a los $\mathrm{LNH}$ en cavidad bucal, estos pueden desarrollarse de forma central en los maxilares o en los tejidos blandos, presentándose como aumentos de volumen difusos y asintomáticos que afectan el vestíbulo bucal, el paladar duro o la encía, su consistencia es dura, se observan eritematosos o violáceos y pueden o no presentar úlceras. Los pacientes portadores de prótesis totales refieren dificultad para ajustarlas, sintiendo mucha presión a la colocación debido a que las prótesis se encuentran en contacto con el aumento de volumen ${ }^{4,5}$.

El objetivo de este trabajo fue la presentación de un caso clínico, donde se expone el diagnóstico, presentación clínica, tratamiento y evolución de un LNH en cavidad oral y la búsqueda de bibliografía para revisar aspectos importantes en el conocimiento de esta entidad.

\section{Reporte del caso}

Se trata de paciente masculino de 80 ańos que presenta como antecedente personal patológico de relevancia diagnóstico de hipertensión arterial sistémica actualmente controlada. El paciente refiriere haber iniciado cuadro clínico con percepción de aumento de volumen en región naso geniana derecha dos meses previos a la consulta posterior a un traumatismo menor en dicha región, por lo que acude con odontólogo particular quien indicó terapia antiinflamatoria y antimicrobiana sin mejoría y lo refiere para valoración con el servicio de cirugía maxilofacial. A la exploración física se observó aumento de volumen en región naso geniana derecha, difusa, no dolorosa a la palpación, indurada (Figura 1). Al interrogatorio, el paciente negó parestesias. Intra oralmente presentó aumento de volumen en región maxilar posterior derecha en zona vestibular de coloración similar a mucosa adyacente, indurada, difusa de base sésil de aproximadamente $2 \mathrm{~cm}$ de diámetro mayor (Figura 2), presentó movilidad grado III en órganos dentarios adyacentes; primero y segundo molar superior derechos.

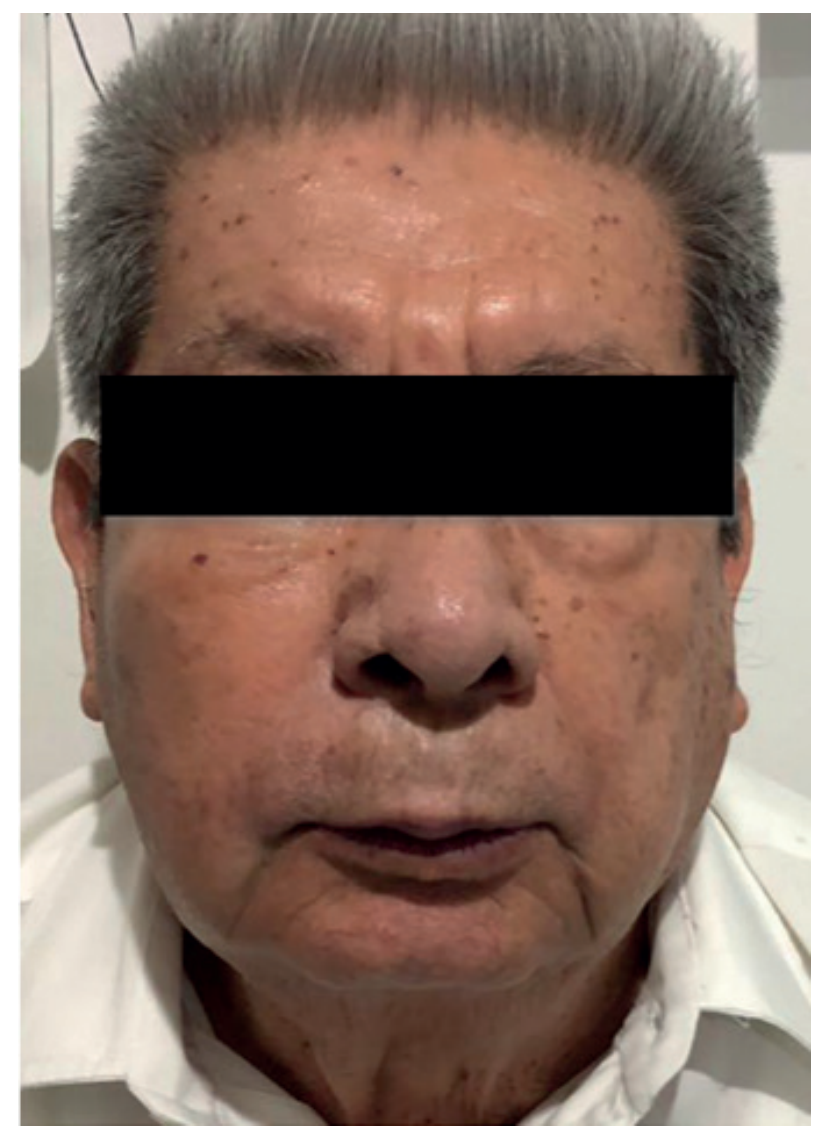

Figura 1. Fotografía clínica extraoral observando aumento de volumen en región naso geniana derecha

Se solicitó tomografía computarizada (TC) simple y contrastada de cabeza y cuello, donde se observó en cortes axiales y coronales lesión hipodensa a hueso que abarca piso de órbita, ocupa porción de seno maxilar e infiltra pared anterior del mismo hacia región de fosa 
cigomática (Figura 3). Se decide realizar toma de biopsia incisional bajo anestesia local, obteniendo tejido de consistencia blanda, friable de color blanquecino, el cual se envía a estudio histopatológico, observando un espécimen formado por linfocitos medianos y grandes con pleomorfismo e hipercromatismo en un tejido conectivo fibroso denso bien vascularizado con hemorragia reciente (Figura 4).

El paciente fue referido al Instituto Nacional de Cancerología de México (INCAN), donde se realizó estudio de inmuno histoquímica con resultados de: cCD-20, bcl-1, bcl-6, CD-10 positivos; NUM-1 positivo; CD3, EBERISH: negativos y un diagnóstico definitivo del infoma no-Hodgkin de células grandes B tipo centro germinal.
El paciente recibió tratamiento en dicho instituto a base de radioterapia dirigida a la zona de la lesión (36Gy) y 6 ciclos de quimioterapia con R-CHOP (rituximab, ciclofosfamida, clorhidrato de doxorubicina (hidroxidaunorubicina), sulfato de vincristina (Oncovin) y prednisona). El paciente respondió favorablemente al tratamiento y a los dos años después del diagnóstico se encuentra libre de lesión y en fase de control (Figuras 5 y 6 ).

Revisión de la literatura. Dentro de Estados Unidos de Norteamérica, en el año 2017 se estimó que más de 650 000 personas vivían con o se encontraban en remisión de $\mathrm{LNH}$, calculando que en ese año habría aproximadamente 74680 casos nuevos y que se producirían cerca de 19 910 muertes a causa de esta enfermedad para el $2018^{6}$.

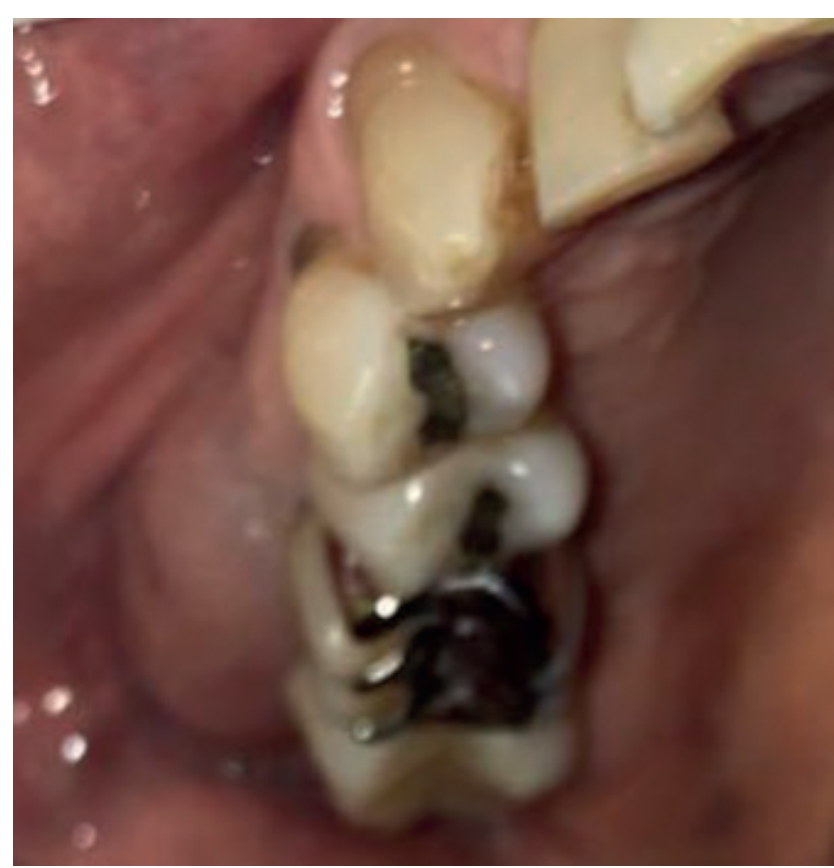

Figura 2. Fotografía clínica intraoral donde se observa aumento de volumen en zona vestibular de la región molar derecha del maxilar

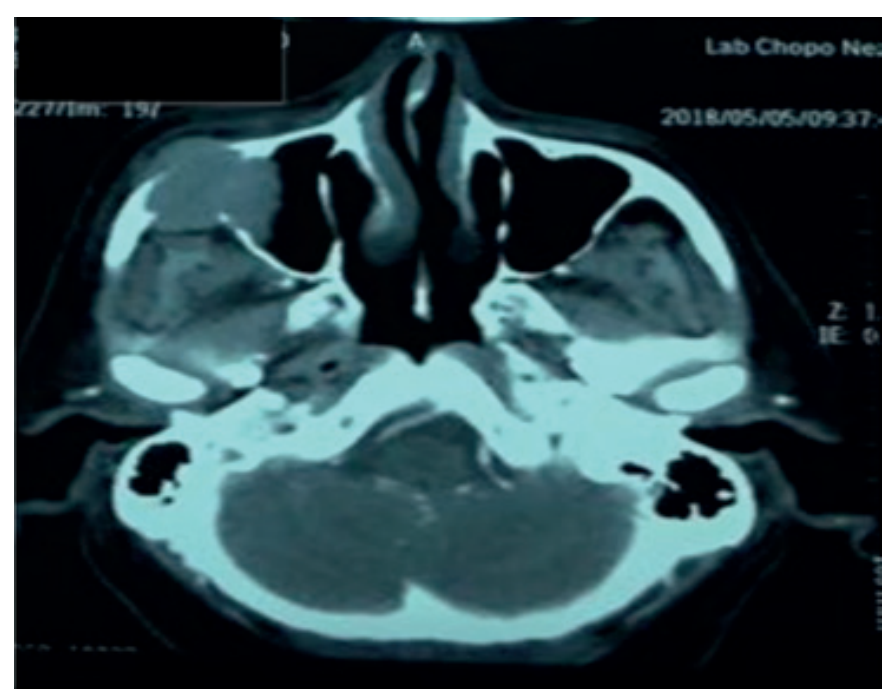

Figura 3. TC corte axial. Se observa lesión hipodensa a hueso que ocupa porción del seno maxilar derecho y destruye pared anterior del mismo 
En México, en el año 2003, esta patología constituyó el tercer tipo de cáncer más frecuente en hombres $(7,8 \%)$, antecedido por el cáncer de piel y próstata. En mujeres, el linfoma fue la sexta causa de cáncer (3,97\%). Asimismo, se ha logrado observar una tendencia al incremento en la frecuencia de los linfomas, ya que en 1997 los casos de linfoma en hombres y mujeres solo correspondían a 7,1 y 2,6\% respectivamente. En el 90\% de los casos, el linfoma no Hodgkin, se presenta en la edad productiva del individuo; entre los 40 y 60 ańos ${ }^{7}$.

Desde el año 2000 hasta 2017 los linfomas han representado la octava causa de muerte por cáncer en México, con incidencia ajustada por edad de 7,8 por cada 100000 habitantes y tasa de mortalidad estandarizada por edad de 3,7 por cada 100,000 habitantes. En un estudio multicéntrico efectuado en México se analizaron 523 casos en 11 hospitales; la mediana de edad de los pacientes fue de 59 años (48-69); donde la proporción de presentación según género masculino: femenino fue de; 1:1; el linaje B representó $89,5 \%$ de los casos contra $10,5 \%$ del linaje T. De acuerdo con la presentación del subtipo se obtuvieron en orden de frecuencia los siguientes datos: linfoma difuso de células grandes B (57\%), linfoma folicular $(14,7 \%)$ y linfoma de linfocitos pequeńos (4\%); el linaje T por subtipo: T/NK (5.4\%) y linfoblástico (2,1\%). Se clasificó enfermedad temprana en 45,1\% (Ann-Arbor I-II) y enfermedad avanzada en $54,7 \%$ (III-IV) ${ }^{8}$. El $80-85 \%$ de los LNH se originan en los linfocitos $\mathrm{B}$, afectan a precursores o a células maduras, observando una superposición entre la leucemia y el linfoma no Hodgkin, porque ambos causan proliferación de linfocitos o sus precursores; $50 \%$ se encuentran

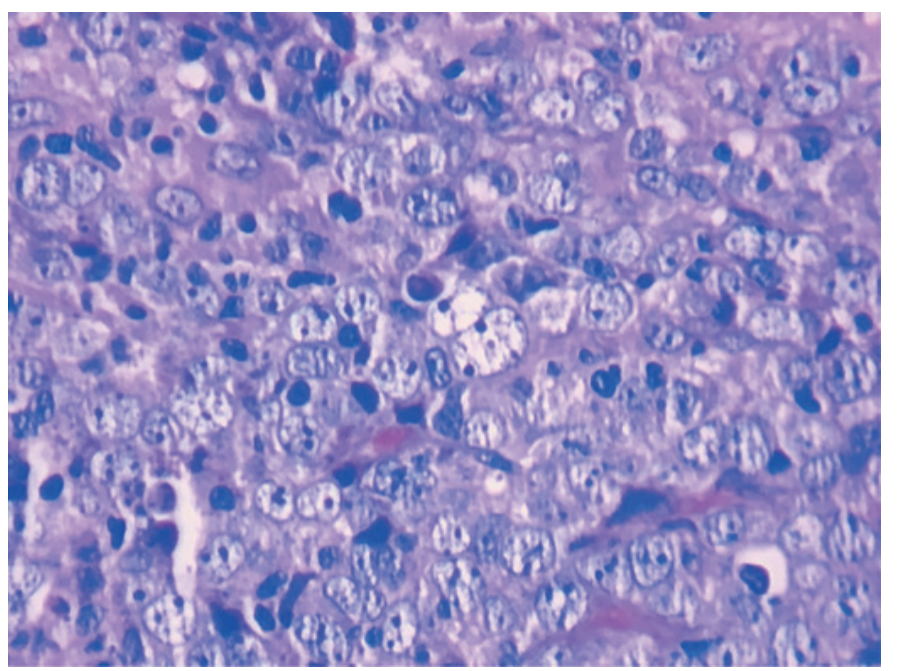

Figura 4. Corte histológico de biopsia tomada de la lesion en fondo de vestíbulo a $40 \mathrm{x}$. Tinción HE donde se observan linfocitos medianos y grandes con pleomorfismo e hipercromatismo en un tejido conectivo fibroso denso bien vascularizado con hemorragia reciente
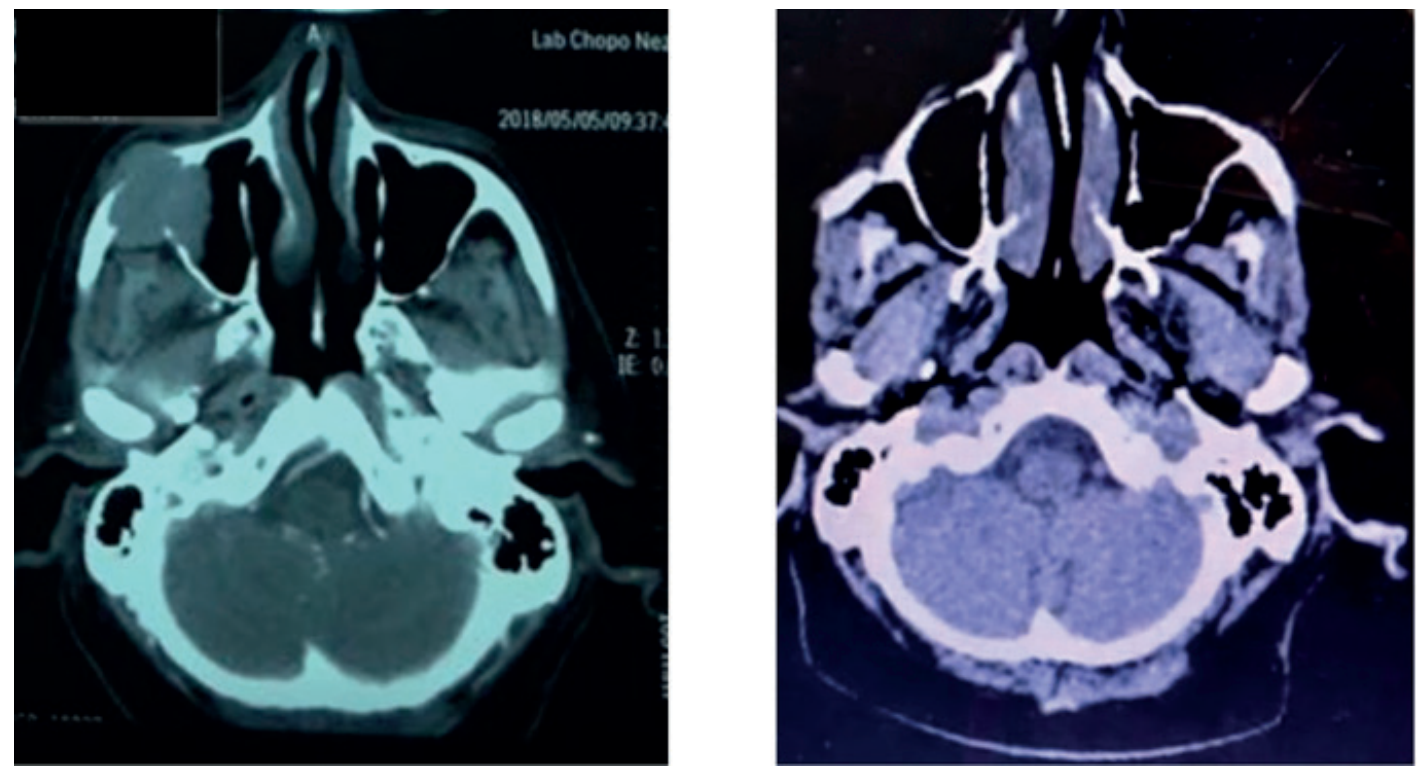

Figura 5. TC comparativas pre y post observando remisión completa de la lesión 


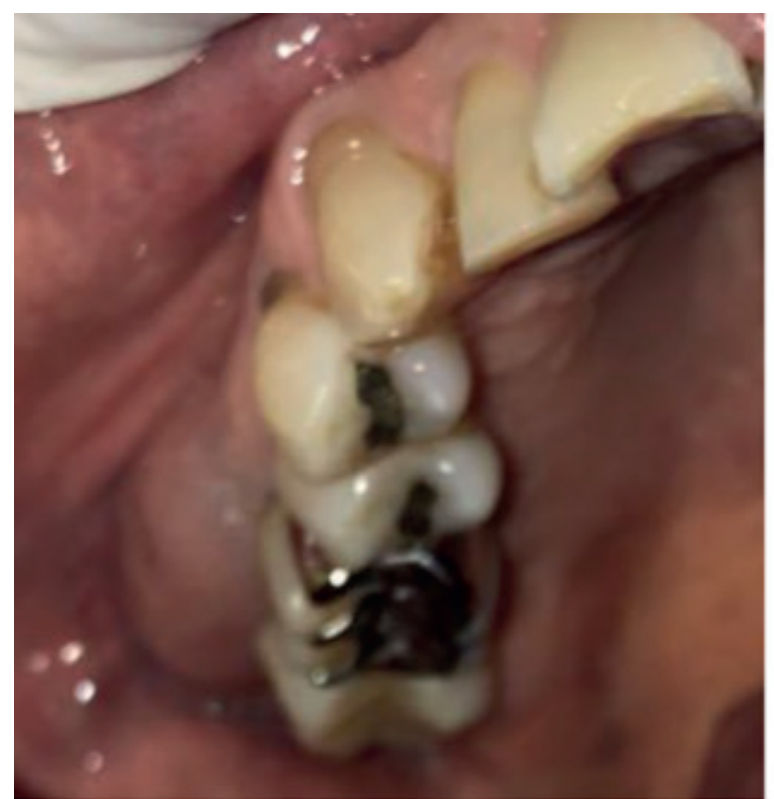

A

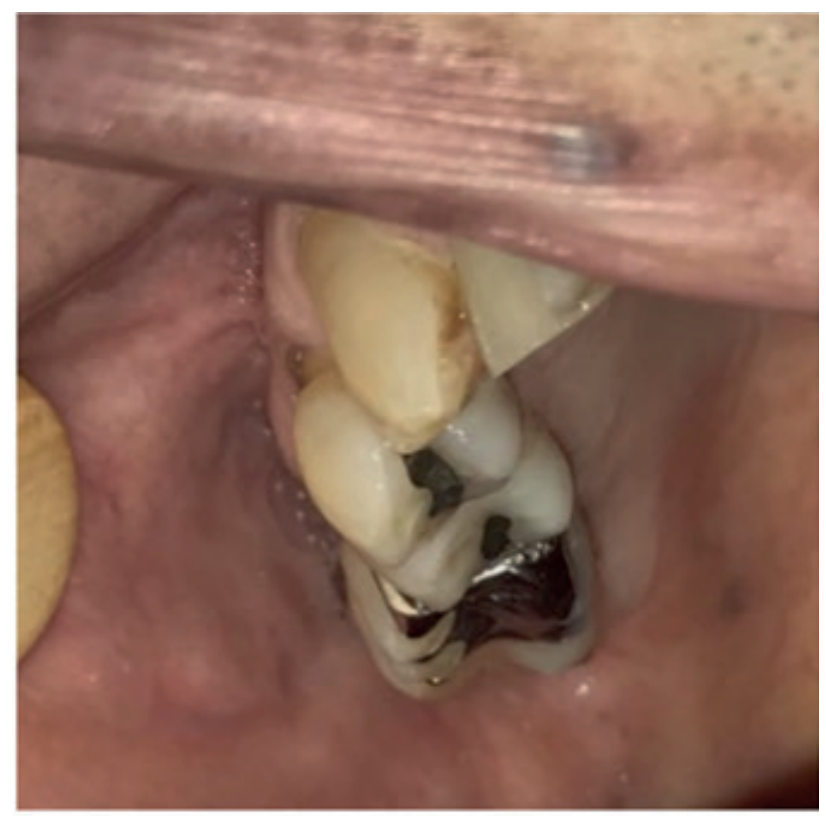

B

Figura 6. A. Aspecto clínico inicial de la lesión. B. Aspecto post-tratamiento observando remisión completa de la lesión.

en pacientes pediátricos y en alrededor de $20 \%$ de los adultos con tipos de linfoma no Hodgkin, puede haber un cuadro similar a la leucemia con linfocitosis periférica y compromiso de la médula ósea ${ }^{7-8}$. Como antecedentes históricos el nombre utilizado para identificar a éstas lesiones, se le atribuye a Samuel Wilks quien en 1865, inmortaliza a su colega Thomas Hodgkin, publicando la primera descripción de enfermedad de Hodgkin, consecuentemente, identificando al grupo de neoplasias linfoides con una variación más heterogénea como linfoma No-Hodgkin. Virchow y Cohnheim en 1864 y 1965 hacen una distinción entre leucemia y linfosarcoma, comparando algunos casos descritos por Hodgkin en $1832^{3}$.

Bilroth en 1871, es el pionero en la conceptualización del linfoma "maligno" y en el año de 1892 Dreshfield, desarrolla los criterios histológicos para la detección del "linfosarcoma", criterios readaptados posteriormente por Roulet en 1930. Gracias a la descripción de Brill y Symmers del linfoma "folicular-gigante" en 1925, se describe a esta entidad como la enfermedad de Brill Symers ${ }^{8}$. Posteriormente, en 1958, Burkitt describe en Africa una variedad de linfoma que hoy lleva su nombre y en 1977 Uchiyama describe lo que se llama linfoma/ leucemia de células $\mathrm{T}$, que se asocia a virus linfotrópico humano (HTLV). Años después, en 1982, el Instituto Nacional de Cáncer de EE.UU publica The Working Formulation, dónde establece una clasificación de pronóstico para LNH la cual se divide en 3 grupos: bajo, medio y alto ${ }^{3}$.

Actualmente, existen alrededor de 6 clasificaciones para linfoma no-Hodgkin, entre las cuales las más utilizadas son las de Rappaport (1966), Dorfman (1974), Kiel (1974) y OMS de trabajo para uso clínico en (1982).
Otra clasificación que agrupa las neoplasias linfoides en general, aparece en 1994 en la revista Blood y posteriormente otra en mayo de 1995, que se publica en The American Journal of Clinical Pathology por Harris, Jaffe y Stein, donde se incluyen entidades no clasificadas entonces como; el "Burkitt Like"; linfomas angiocéntricos; linfoma tipo MALT (tejido linfoide asociado a las mucosas) y GALT (tejido linfoide asociado al tracto gastrointestinal); linfoma esplénico de zona marginal; linfoma anaplásico; linfoma tipo células NK (natural killers); linfoma periférico de células $T$ y ahora un tipo de linfoma de Hodgkin distinto de la enfermedad de Hodgkin ${ }^{3}$.

La etiología en la mayoría de los casos es desconocida, en algunos casos la evidencia sugiere una asociación con leucemia de linfocitos T, virus de Epstein Barr, virus de hepatitis C y VIH (El linfoma no Hodgkin es la segunda neoplasia maligna, en orden de frecuencia en pacientes que presentan infección por VIH, los reordenamientos del gen C-myc son característicos de algunos linfomas asociados con SIDA) ${ }^{\text {? }}$.

Factores de riesgo: estados de inmunodeficiencia congénita o adquirida, enfermedades autoinmunes (síndrome de Sjögren, tiroiditis, enfermedad Celiaca), con antecedentes de infecciones virales (Epstein Barr, HTVL-1), infección por H. Pylori, exposición a sustancias químicas y tratamiento oncológico previo para linfoma Hodgkin 9. Factores predisponentes: genéticos, inmunodeficiencia congénita o adquirida e inmunosupresión farmacológica crónica ${ }^{9-10}$.

El diagnóstico clínico se basa en la presencia de fiebre, diaforesis, pérdida de peso y síndrome infiltrativo, encontrado a la exploración clínica: adenopatías, esplenomegalia y hepatomegalia, la mayor parte de los pacientes 
con LNH presentarán adenopatías de crecimiento progresivo, indoloras de localización cervical, axilar o inguinal, las localizaciones extra ganglionares primarias más frecuentes comprenden: estómago, intestino delgado, piel y cerebro(con presencia de síntomas neurológicos o bien datos clínicos por compresión medular como: parestesias y paresias) ${ }^{11-12}$.

La boca representa un sitio extra ganglionar poco común para neoplasias de tejido linfoide. Los linfomas de la boca, en cuanto a neoplasias primarias, son exclusivamente $\mathrm{LNH}$. Los tipos histológicos de estos linfomas tienen frecuencia y nomenclaturas variables, en función de varios sistemas de clasificación ${ }^{12}$. Clínicamente, por lo general son lesiones de consistencia blanda, con ulceración de la mucosa que lo recubre, se aprecia movilidad dentaria, perdida de hueso alveolar y ausencia de sintomatología. Los linfomas de cavidad bucal representan el $4 \%$ de los casos de cáncer en cavidad bucal ${ }^{13}$. En el área de cabeza y cuello los linfomas se observan dentro de los ganglios linfáticos regionales y dentro de regiones linfoideas extra linfáticas en áreas que se conocen como tejido linfoideo asociado a intestino o mucosa. Dentro de la cavidad bucal el tejido linfoideo lo representa de manera principal el anillo de Waldeyer y en otras partes de la cavidad bucal se representa como tejido linfoide no encapsulado, en cuanto a su aparición en los maxilares, es más frecuente encontrar este tipo de lesiones en el maxilar que en la mandíbula ${ }^{13-15}$.

Los signos y síntomas que sugieren la presencia de un linfoma en la región de cabeza y cuello son: alteraciones de la sensibilidad, movilidad dental, aumento de volumen, dolor dental sin causa aparente, aumento de tamaño localizado en tejido óseo. Debido a estos signos y síntomas, se consideran como diagnósticos diferenciales; absceso dental, enfermedad periodontal o hiperplasia fibrosa inflamatoria. En el $10-20 \%$ de los casos, no pueden encontrar signos imagenológicos de tejido óseo involucrado en LNH, por lo que los hallazgos pueden ser radiológicamente inespecíficos observando zonas de destrucción ósea difusa, defectos óseos localizados u horizontales, parecidos a los encontrados en casos de periodontitis ${ }^{16-18 .}$

Se debe complementar el diagnóstico realizando una biopsia representativa de la zona tumoral o una revisión del material disponible, para diagnóstico histopatológico, posteriormente se debe realizar inmunohistoquímica con el fin de caracterizar el tipo celular, la tomografía computarizada por emisión de positrones (PET TC) se puede efectuar para evaluar respuesta al tratamiento ${ }^{19}$. En el grupo de LNH agresivos, debe establecerse el índice pronostico internacional (IPI) que valora: edad, etapa de Ann Arbor, desempeño físico (ECOG), DHL y sitios de infiltración extra nodal. En el grupo de LNH indolente debe establecerse el índice pronóstico internacional para linfoma folicular (FLIPI) que valora: edad, etapa de Ann Arbor, desempeño físico (ECOG), Hb y número de sitios ganglionares, en caso de estar accesible realizar inmunohistoquimica bcl-2, bcl-6 y/o bcl-2/IgH ${ }^{10,14}$.
La determinación del pronóstico del LNH puede efectuarse utilizando los sistemas representados a continuación, en donde se toma en cuenta la localización de la lesión y las características del paciente.

Tabla 1. Estadificación de los linfomas no Hodgkin de acuerdo a Ann Arbor ${ }^{2,16}$

\begin{tabular}{cl}
\hline Estadio & \multicolumn{1}{c}{ Definición } \\
\hline I & $\begin{array}{l}\text { Involucra una sola región ganglionar o un solo órgano o } \\
\text { sitio extralinfático (IE). }\end{array}$ \\
II & $\begin{array}{l}\text { Dos o más regiones ganglionares a un solo lado del } \\
\text { diafragma. }\end{array}$ \\
& $\begin{array}{l}\text { Involucración de un solo órgano o sitio extra linfatico y } \\
\text { uno ó más regiones linfoides a un solo lado del diafrag- } \\
\text { ma (IIE). Puede indicarse el número de regiones nodales } \\
\text { involucradas (IIE })_{3} \text { ). }\end{array}$ \\
& $\begin{array}{l}\text { Involucración de regiones de ganglios linfáticos en } \\
\text { ambos lados del diafragma. Pudiendo estar acompañado } \\
\text { por involucración localizada de un sitio u órgano extra } \\
\text { nodal ( IIIE) o bazo ( IIIS) o ambos ( IIIES). }\end{array}$ \\
& $\begin{array}{l}\text { Involucración difusa o diseminada de uno o más órga- } \\
\text { nos extranodales a distancia con o sin involucración de } \\
\text { ganglios linfáticos agrandados. }\end{array}$ \\
\hline
\end{tabular}

En presencia de uno o más síntomas sistémicos: Fiebre mayor a $38^{\circ} \mathrm{C}$, sudoración nocturna y/o pérdida de peso mayor del $10 \%$ en los últimos 6 meses, se agrega $\mathrm{B}$ a la clasificación. De no existir ninguno se agrega A.

Tabla 2. Indice Pronóstico Internacional (IPI) en linfoma no Hodgkin ${ }^{21,22}$

\begin{tabular}{cc}
\hline $\begin{array}{c}\text { Número de Factores de } \\
\text { riesgo }\end{array}$ & CATEGORIA IPI \\
\hline $0-1$ & Bajo \\
2 & Bajo intermedio \\
3 & Alto intermedio \\
4 ó 5 & Alto \\
\hline
\end{tabular}

Un punto por cada una de las variantes siguientes:

1. Edad mayor de 60 años

2. Puntuación de 2 o más en la escala Eastern Cooperative Oncology Group (ECOG).

3. Estadio III ó IV ( Ann Arbor)

4. Sitios extranodales ( más de 1 )

5. Deshidrogenasa láctica (DHL) elevada.

\section{Discusión}

El LNH en cavidad oral es poco frecuente, aún más si se trata de presentación primaria extra ganglionar. Freeman et al. ${ }^{11}$ reportaron que, de 1467 casos de linfomas extra ganglionares estudiados, solo un $2 \%$ se encontraba en cavidad oral. Por otra parte, Guevara-Canales et al $l^{12}$ publicaron que, de 3513 casos de linfoma, el $4 \%$ correspondía a lesiones en nivel de cavidad oral y región maxilofacial. 
En este artículo, se presenta un caso de LNH de células grandes B, subtipo histológico, que, según la literatura, es considerado el más frecuente. Yin et al. ${ }^{20}$ reportaron que el $78 \%$ de LNH corresponde a linfomas de células B mientras que Neville et al. ${ }^{17}$ mencionan que en Europa y EE. UU esta estimación corresponde hasta al $85 \%$ de las neoplasias linfoides.

De acuerdo con lo reportado en la literatura, la edad promedio en la que este tipo de lesiones se presenta, es variable. La mayoría de los autores coinciden en que estas neoplasias se presentan con mayor frecuencia entre los 40 y 70 ańos y que existe una leve predominancia en pacientes de género masculino. Van der Waal et al. ${ }^{21}$ quienes revisaron 40 casos de $\mathrm{LNH}$ primario de la cavidad oral, reportaron como edad promedio los 59 años, Shah et al. ${ }^{22}$ mencionan una media etaria de 42,6 años, mientras que Guevara-Canales et al. ${ }^{12}$ publican que el $27,81 \%$ de una muestra de 151 linfomas en cavidad oral, se presentaron en pacientes de 61 a 70 años, siendo el $53,64 \%$ correspondiente a individuos de género masculino.

La evidencia científica, indica que el linfoma no Hodgkin afecta principalmente a pacientes inmunosuprimidos. Contreras et al. ${ }^{23}$ mencionan que los pacientes con mayor riesgo de presentar un linfoma agresivo, como lo es el LNH, son aquellos; infectados por el virus de inmunodeficiencia adquirida (VIH), pacientes que han recibido trasplantes de órganos o aquellos con desordenes inmunológicos. Asimismo, Varun et al. ${ }^{24}$ reportan que el riesgo para $\mathrm{LNH}$ es 60 veces mayor en los pacientes $\mathrm{VIH}$-positivos.

El caso aquí reportado, coincide con la epidemiología documentada en la literatura en cuanto al género, más no así, en rango de edad y factores de riesgo, habiendo diagnosticado al paciente a los 80 años y sin que éste contara con factores de riesgo relevantes.

Se destaca la importancia de documentar este caso, al describir una entidad poco frecuente, como lo es el linfoma no Hodgkin en cavidad oral, tratándose además de una presentación inusual como lesión primaria en un paciente sin inmunocompromiso, es importante realizar consulta a un experto ya que puede ser tratado erróneamente como una patología de naturaleza odontógena por su analogía clínica.

En conclusión es necesario que los odontólogos generales y los especialistas, cuenten con los conocimientos necesarios para poder detectar de manera precoz neoplasias como el linfoma no-Hodgkin en cavidad oral, considerando, también, la posibilidad de que el LNH se manifieste en boca como una lesión primaria, lo cual puede significar el inicio de una progresión de la enfermedad hacia otros nódulos linfáticos y estructuras anatómicas. Teniendo conocimiento sobre los parámetros clínicos podemos identificar de manera temprana $\mathrm{LNH}$ analizando lesiones de consistencia blanda en región oral, con ulceración de la mucosa que lo recubre, que cuenten con movilidad dentaria, perdida de hueso alveolar y ausencia de sintomatología sistémica ó sumado a parámetros clínicos sistémicos como lo son ganglios que no regresen a su tamaño posterior a 14 días de tratamiento antibiótico y antiinflamatorio, localización supraclavicular, consistencia dura, ausencia de dolor, historia reciente de síntomas en oídos, nariz y garganta, fiebre mayor a $38^{\circ} \mathrm{C}$, sudoración nocturna y/o pérdida de peso mayor del $10 \%$ en los últimos 6 meses, los datos anteriores en conjunto con resultados de laboratorio: biopsia con infiltración de celularidad asociada, alteración en DHL, leucocitosis, hiperuricemia, hiperkalemia, hiperfosfatemia y creatinina elevada.

Un diagnóstico temprano de LNH, aumentará las probabilidades de un buen pronóstico.

\section{Referencias bibliográficas}

1. Morton LM, Wang SS, Cozen W, Linet MS, Chatterjee $\mathrm{N}$, Davis $\mathrm{S}$ et al. Etiologic heterogeneity among non-Hodgkin lymphoma subtypes. Blood. 2008;112(13):51505160. DOI: 10.1182/blood-2008-01-133587.

2. Instituto Mexicano del Seguro Social. Guía de referencia para diagnóstico de LNH. CENETEC SALUD. [Internet]. 2018 [Citado el 26 de enero 2020]. Accesible en: http://www.cenetec.salud.gob.mx/descargas/gpc/ CatalogoMaestro/447_GPC_Linfoma_no_Hodgkin/ GER_Linfoma_no_Hodgkin.pdf=

3. Müller AMS, Ihorst G, Bertelsmann R, Engelhardt M. Epidemiology of non-Hodgkin's lymphoma (NHL): trends, geographic distribution, and etiology. Ann Hematol. 2005;84:1-12. DOI: 10.1007/s00277-0040939-7

4. Deng D, Wang Y, Liu W, Qian Y. Oral and maxillofacial non-Hodgkin lymphomas: Case report with review of literature. Medicine (Baltimore). 2017;96(35):e7890. DOI: 10.1097/MD.0000000000007890.

5. Labardini JR, Cervera E, Corrales C, Balbuena M, Barbosa AA, Espinosa JR et al: Linfoma No Hodgkin. Rev Inst Nac Cancerol (Mex). 2011;139-152.

6. American Cancer Society. Cancer Facts \& Figures 2018. Atlanta: American Cancer Society [Internet]. 2018 [Citado el 02 de febrero 2019]. Accesible en: https:// www.cancer.org/content/dam/cancer-org/research/cancer-facts-and-statistics/annual-cancer-facts-and-figures/2018/cancer-facts-and-figures-2018.pdf.

7. SINAIS/SINAVE/DGE/SALUD Perfil epidemiológico de los tumores malignos en México. [Internet] México, D. F: Secretaría de Salud; 2011.197 p. Disponible en: https://epidemiologiatlax.files.wordpress.com/2012/10/p_ epi_de_los_tumores_malignos_mc3a9xico.pdf

8. Lakhtakia, R., \& Burney, I. A historical tale of two lymphomas: part II: non-Hodgkin lymphoma. Sultan Qaboos Univ Med J. 2015;(3):317-21. DOI:10.18295/ squmj.2015.15.03.003.

9. Bassig BA, Lan Q, Rothman N, Zhang Y, Zheng T. Current understanding of lifestyle and environmental factors and risk of non-hodgkin lymphoma: an epidemiological update. J Cancer Epidemiol. 2012;2012:978930.

10. Morton LM, Slager SL, Cerhan JR, Wang SS, Vajdic CM, Skibola CF et al. Etiologic heterogeneity among non-Hodgkin lymphoma subtypes: the interlymph 
non-Hodgkin lymphoma subtypes project. J Natl Cancer Inst Monogr. 2014;(48),130-44. DOI: 10.1093/jncimonographs/lgu013.

11. Freeman C, Berg JW, Cutler SJ. Occurrence and prognosis of extranodal lymphomas. Cancer.[Internet] 1972 [citado 5 de setiembre de 2019];29:25260. Disponible en: https://acsjournals.onlinelibrary. wiley.com/doi/epdf/10.1002/1097-0142\%2819 $7201 \% 2929 \% 3 \mathrm{~A} 1 \% 3 \mathrm{C} 252 \% 3 \mathrm{~A} \% 3 \mathrm{AAID}-\mathrm{CN}$ CR2820290138\%3E3.0.CO\%3B2-\%23 DOI: 10.1002/1097-0142(197201)29:1<252::AID-CNCR2820290138>3.0.CO;2-\%23.

12. Guevara-Canales JO, Morales-Vadillo R, Sacsaquispe-Contreras SJ, Barrionuevo-Cornejo C, Montes-Gil J, Cava-Vergiú CE et al. Malignant lymphoma of the oral cavity: and the maxilofacial region: overall survival prognostic factor. Med Oral Patol Oral Cir Bucal. 2013;18(4):e619-26. DOI:10.4317/medoral.18903.

13. Perry, A. M., Diebold, J., Nathwani, B. N., MacLennan, K. A., Müller-Hermelink, H. K., Bast, M., Boilesen et al. Non-Hodgkin lymphoma in the developing world: review of 4539 cases from the International non-Hodgkin lymphoma classification project. Haematologica. 2016;101(10),1244-1250. DOI: 10.3324/haematol.2016.148809.

14. Hachem A, Gartenhaus RB. Oncogenes as molecular targets in lymphoma. Blood. 2005;106(6),1911-1923. DOI: 10.1182/blood-2004-12-4621.

15. Mosqueda-Taylor A; Meneses-García A; Zarate-Osorno A. Ruiz-Godoy LM, Ochoa-Carrillo FJ, Mohar-Betancourt A. Angiocentric lymphomas of the palate: Clinico-pathological considerations in 12 cases. J Oral Pathol Med 1997;26:93-97.

16. Freedman A; Nadler L. Linfomas Malignos. Harrison, principios de medicina interna. 13 ed. Madrid: Editorial Interamericana McGraw Hill;1994. Vol. II. P. 20202054.
17. Neville B, Damm D, Allen C, Chi A. Oral and maxillofacial pathology. 4ta ed. Philladelphia: Saunder; 2015. pp. 517-521.

18. Moin A, Shetty AD. Management of Non-Hodgkin's Lymphoma in Maxillofacial Region with Chemotherapy. Ann Maxillofac Surg. 2017;7(2):312-315. DOI: 10.4103/ams.ams_85_17.

19. Hennessy BT, Hanrahan EO, Daly PA. Non-Hodgkin lymphoma: an update. Lancet Oncol. 2004;5:341-53. DOI: 10.1016/S1470-2045(04)01490-1.

20. Yin HF, Jamlikhanova V, Okada N, Takagi M. Primary natural killer/T-cell lymphomas of the oral cavity are aggressive neoplasms. Virchows Arch. 1999;435:400-6.

21. Van der Waal RI, Huijgens PC, van der Valk P, van der Waal I. Characteristics of 40 primary extranodal non-Hodgkin lymphomas of the oral cavity in perspective of the new WHO classification and the International Prognostic Index. Int J Oral Maxillofac Surg. 2005;34:391-5. DOI: 10.1016/j.ijom.2004.08.009.

22. Shah GH, Panwar SK, Chaturvedi PP, Kane SN. Isolated primary extranodal lymphoma of the oral cavity: A series of 15 cases and review of literature form tertiary care cancer center in India. Indian J Med Paediatr Oncol. 2011;32(2):76-81. DOI: 10.4103/0971-5851.89776.

23. Contreras E, Bagán JV, Lloria E, Borja A, Millán M.A., Jiménez Y. Linfoma no-Hodgkin intraoral. Presentación de 4 casos clínicos. Acta Otorrinolaringol Esp. 2001;52:609-614. DOI: 10.1016/S00016519(01)78255-6.

24. Varun BR, Varghese NO, Sivakumar TT, Joseph AP. Extranodal Non-Hodgkin's Lymphoma of the Oral Cavity: A Case Report. Iran J Med Sci. 2017;42(4):407-411. 